\title{
A NEW MEASURING FIXATOR DESIGN FOR HUMERAL SUPRACONDYLAR CORRECTIVE OSTEOTOMIES IN CHILDREN
}

\author{
ShuO-SueI Hung ${ }^{1}$, MING-YIH LEE ${ }^{2}$, CHIN-HSIUNG Hsu², \\ WEN-DA TZAI ${ }^{2}$, ZHON-LIAO LEE ${ }^{3}$
}
${ }^{1}$ Department of Orthopedic Surgery, Chang Gung Memorial Hospital and St. Paul's Hospital, ${ }^{2}$ Graduate Institute of Mechanical Engineering, Chang Gung University, ${ }^{3}$ Department of Orthopedic Surgery, Chang Gung Memorial Hospital and Children's Hospital, Taoyuan, Taiwan

\begin{abstract}
Treatment for bone deformities secondary to malunited fractures usually require corrective osteotomies, and high incidence of surgical failure rates have been reported. Inaccurate corrections and loss of reductions are among the most common causes, and these technical errors ought to be improved [1-2]. A new measuring fixator for humeral supracondylar corrective osteotomies in children was designed and used in clinical application. Several surgical trials were performed in vitro using rapid prototype models to simulate different bony deformities. Two boys who had sustained cubitus varus following malunited humeral supracondylar fracture were performed using proposed measuring fixator. The results of the clinical trial show that the angle of correction was accurate, and the procedure could be carried out by the surgeon himself without additional help, with an average of 1.3 penetration of the physis per each fixating Kirschner wire ( 8 times in $6 \mathrm{~K}$-wires). The surgical time was reduced, as well as the radiation exposure.
\end{abstract}

Biomed Eng Appl Basis Comm, 2003 (February); 15: 27-31.

Keywords: Supracondylar corrective osteotomy, Measuring fixator, Surgical tool

\section{INTRODUCTION}

Cubitus varus is one of the most common complication following humeral supracondylar fracture in children, and the incidences reported varies from $9 \%$ to $57 \%$ [3-5]. Some authors suggest that malunion with medial tilting of the distal fragment may be the main cause leading to this deformity, however, rotation of the distal fragment[6], as well as physeal injury[7],

Received: Jan. 8, 2003; Accept: Feb. 12, 2003

Correspondence: Ming-Yih Lee, Ph.D., Professor Department of Mechanical Engineering,

Chang Gung University, 259 Wen-Hwa 1st Road,

Kwei-Shan, Tao-Yuan, Taiwan

E-Mail: leemiy@mail.cgu.edu.tw may also contribute in other situations. These patients are clinically free of functional limitation, and often come to help for cosmetic purpose due to varus deformity of the elbows. There are seldom complaints of pain or weakness[8].

Several different surgical procedures have been developed in the past with variable results[1,8-10], and they all involve corrective osteotomies. The postoperative complications reported include nerve injury, brachial artery aneurysm, pin tract infection, elbow stiffness, or scarring[5]. However, the most commonly encountered is the recurrence of cubitus varus due to loss of correction, which could be as high as $46 \%$, and the leading causes have been suspected being such as physeal injuries or technical errors[1-2]. To minimize such complications, every single step during the operation must be proceeded carefully. However, without an appropriate surgical tool, surgical procedures may be- 
come difficult and inaccurate

In this paper, design of a handy measuring fixator for corrective osteotomy was reported, which will not only allow the surgeon to obtain an adequate angle of correction, but also serves as a temporary fixator to permit an easier and more accurate fixation of the osteotomy site. Several rapid prototype models of elbow and hip deformities were used for in vitro trials of the device. Surgeries have been performed with this device, and its clinical applicability is discussed.

\section{MATERIALS AND METHODS}

The first step to a successful result during the surgery of valgus closing wedge osteotomy is to obtain an adequate angle of correction, which is usually difficult to determined and very often underestimated intraoperatively. The second obstacle that a surgeon may encounter is to maintain the position of bone fragments prior to fixation. In general, positioning reconfirmation under fluoroscope is required. To minimize the exposure to radiation, a single lateral $\mathrm{K}$-wire from the lateral condyle is usually applied to replace manual holding of the fragment, and if the position is acceptable, additional one or two K-wires will be applied in situ, provided that the fragment has not rotated during this procedure while changing the images from anteroposterior view to a lateral view. Otherwise, repositioning and reconfirmation will have to be repeated. Similarly, if the initial position is not appropriate, readjustment is necessary for the fragment, as well as the fixation of lateral K-wire, and multiple physeal penetration may have resulted.

To confront the above-mentioned problems, a device was designed in-house and intended to allow the surgeons, first of all, to achieve an accurate angle of osteotomy, and with the same device, to obtain a temporary stability of the fragments during fluoroscopic
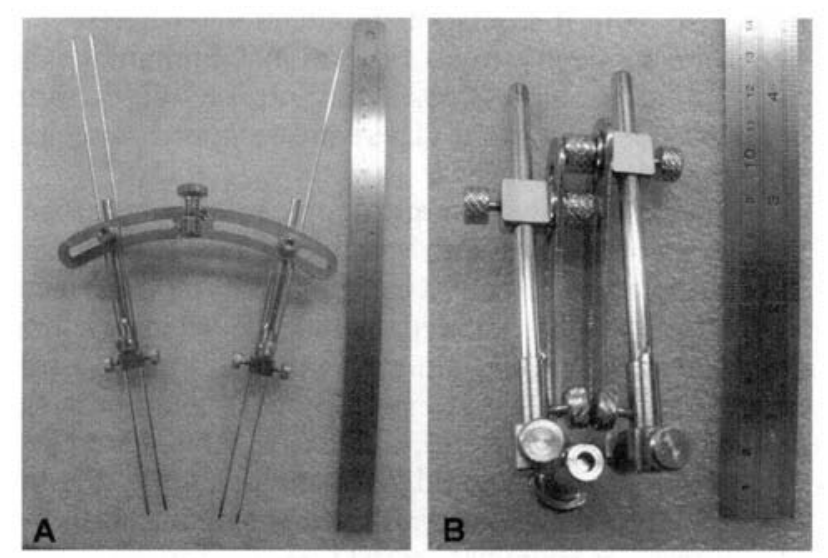

Fig. 1A-View of the device when assembled with $K$ wired inserted over the two arms.1B-View of the device not in use when folded. evaluation before final fixation (patent pending). As shown in Fig. 1A, this stainless steel measuring fixator comprises of a semicircular frame with a central slot, and two arms that slide along the slot with range of motion of 60 degrees of circumference $\left(30^{\circ}\right.$ on each arm). These two arms are connected indirectly onto the bone through two K-wires, and can be used as joysticks for manipulation of the bone fragments. Higher dexterity in manipulation is designed in terms of rotation and translation between the two arms, to compensate with different surgical scenarios. This device is durable, and can be reassembled to a fairly compact size during storage or autoclaving as shown in Fig. 1B.

This measuring fixator was designed using computer aided design / computer aided manufacture (CAD/CAM) technologies. With Solid Works $\$$ package, each component of this device was created individually (Fig. $2 \mathrm{~A}$ ). After that, mechanical links were assembled together for kinematic simulation under the Working Model@ software (Fig. 2B). In this studies, three different prototypes were designed, and the final device was decided through numerous modifications according to the surgeons opinions.

\section{SURGICAL PROCEDURES}

The patient is placed in supine position under standard sterile draping and surgical exposure. After careful preoperative planning, the device is set at the necessary angle, and since there is a residual angle of 5 degrees formed by the two arms at the closest distance due to structural limitation, the same amount of adjustment should be added initially to ensure an adequate correction angle (Fig. 3A). The device is then placed to the osteotomy site, and fixated through the two tunnels on each arm with $\mathrm{K}$-wires. The device is then removed, leaving the $\mathrm{K}$-wires oriented to the preplanned cutting planes, which subsequently serve as guides for performing osteotomy (Fig. 3B).

During surgery, bone resection is done with a small bone saw under well protection, and the device
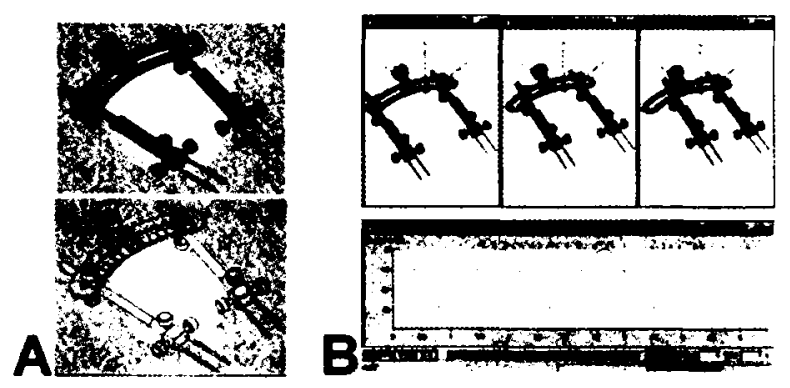

Fig. 2A-CAD design of the device by Solid Works@. 2B-Motion simulation of the device by Working Model@ 


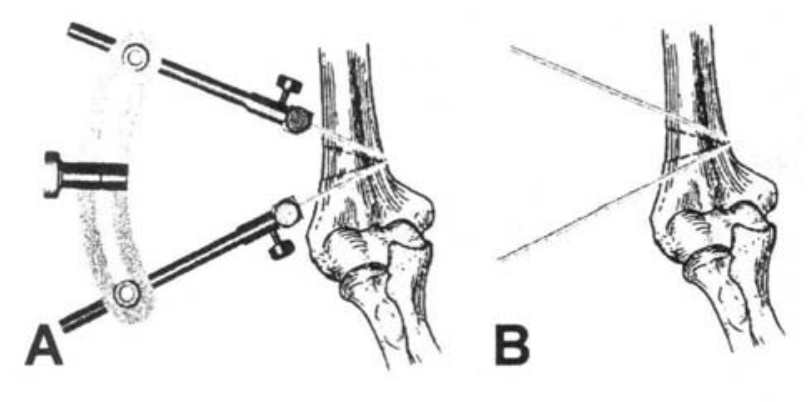

Fig. 3A-The device is adjusted to the angle of osteotomy, and Kirschner wires are inserted through the tunnels over each arm. 3B-The device is dismounted, leaving the Kirschner wires as guides for bone cuts
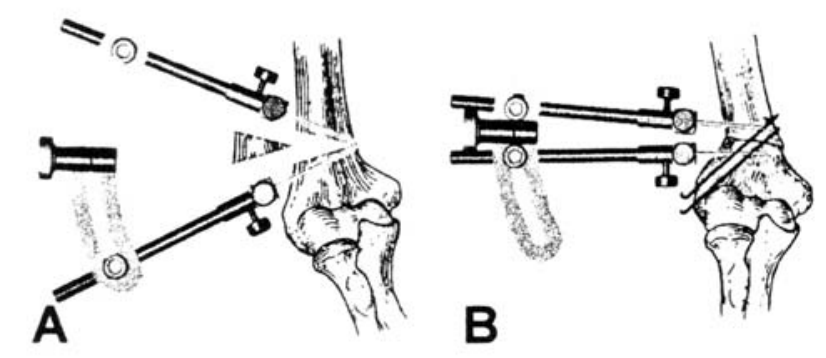

Fig. 4A-The device is repositioned onto the Kirschner wires after osteotomy. 4B-The bone fragements are brought together by manipulating the arms, and fixation is performed.

is mounted back onto the $\mathrm{K}$-wires. The bone fragments are approximated after releasing the locking knobs that connect the arms to the frame, and relocked at the proper position (Fig. 4A). The osteotomy site can be maintained temporarily at a good stability for fluoroscopic examination at this moment, and readjusted easily by releasing the knobs, without reapplication of the $\mathrm{K}$-wires (Fig. 4B). When the position of the fragments are reconfirmed, final fixations can be achieved with additional crossed or parallel K-wires.

\section{RESULTS}

Before clinical trial, we have performed two laboratory tests on the rapid prototyping models, one of the hip and the other one of the elbow, and also on a cadaveric humeral bone with success. Two clinical cases were carried out, and the clinical case presented here was a 5 year old boy who had sustained left elbow injury about 3 years priorly, and cubitus varus resulted following closed reduction and long arm casting. His carry angle was 30 degrees varus over the lesion side, and 5 degrees valgus over the contralateral side, there-

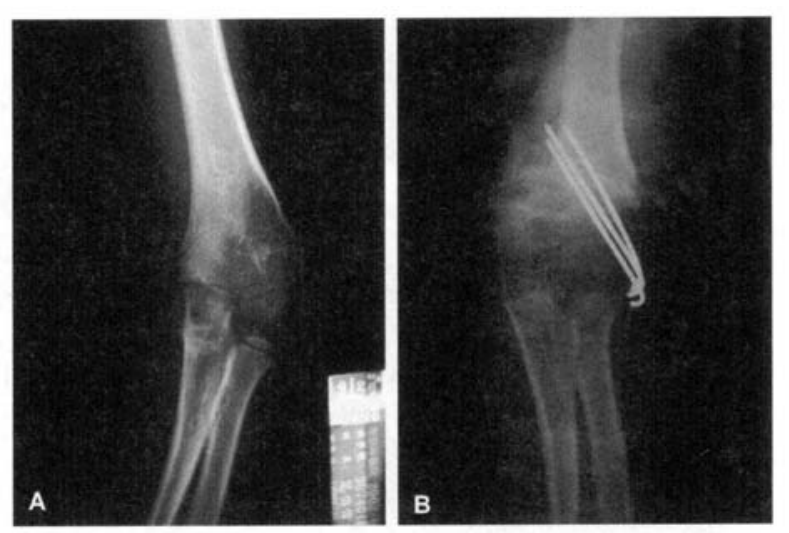

Fig. 5A-Preoperative $X$-ray showing a varus carry angle. 5B-Postoperative $X$-ray film with multiple $K$ wire fixations.

fore, the planned angle of correction was set to be 35 degrees valgus. (Fig. 5A)

Due to good stability obtained by the device, fixation was performed easily without any help of additional assistance (Fig. 6A-B). Three pieces of Kirschner wires were used, and a total of four physeal penetrations were made on this patient's humeral condyle. Intraoperative fluoroscopic evaluation was performed (Fig. 7). The postoperative anteroposterior view is shown on figure $5 \mathrm{~B}$.

\section{DISCUSSIONS}

Corrective osteotomy is the only effective method for correction of established malunited fracture or bone deformity[11-12]. Among the several methods of osteotomies, valgus closing wedge is a simple and promising choice for cubitus varus deformity following malunited humeral supracondylar fracture $[1,13]$.

Even with meticulous preoperative planning, several pitfalls must be overcome during the operation to ensure a successful result. Precise osteotomy is one of the most critical influencing factors, and both lateral wedging and anteroposterior wedging must be taken into account when performing such procedure. Loss of reduction is another main cause of failure, and this can occur either intraoperatively or postoperatively[14]. Fixations are recommended as minimal as possible to avoid multiple penetrations of the cortex and physis, but rotation is inevitable with only a single $\mathrm{K}$-wire fixation as the surgeon would usually apply, during the process of reconfirmation under fluoroscopy and evaluation for the gross elbow range of motion. Lastly, even during the final stage of fixation, either chosen to be with K-wires, screws, or even plates, loss of reduction is still possible without a suitable bone holder[15]. With proposed innovative measuring fixator, the above mentioned problems can be solved eas- 

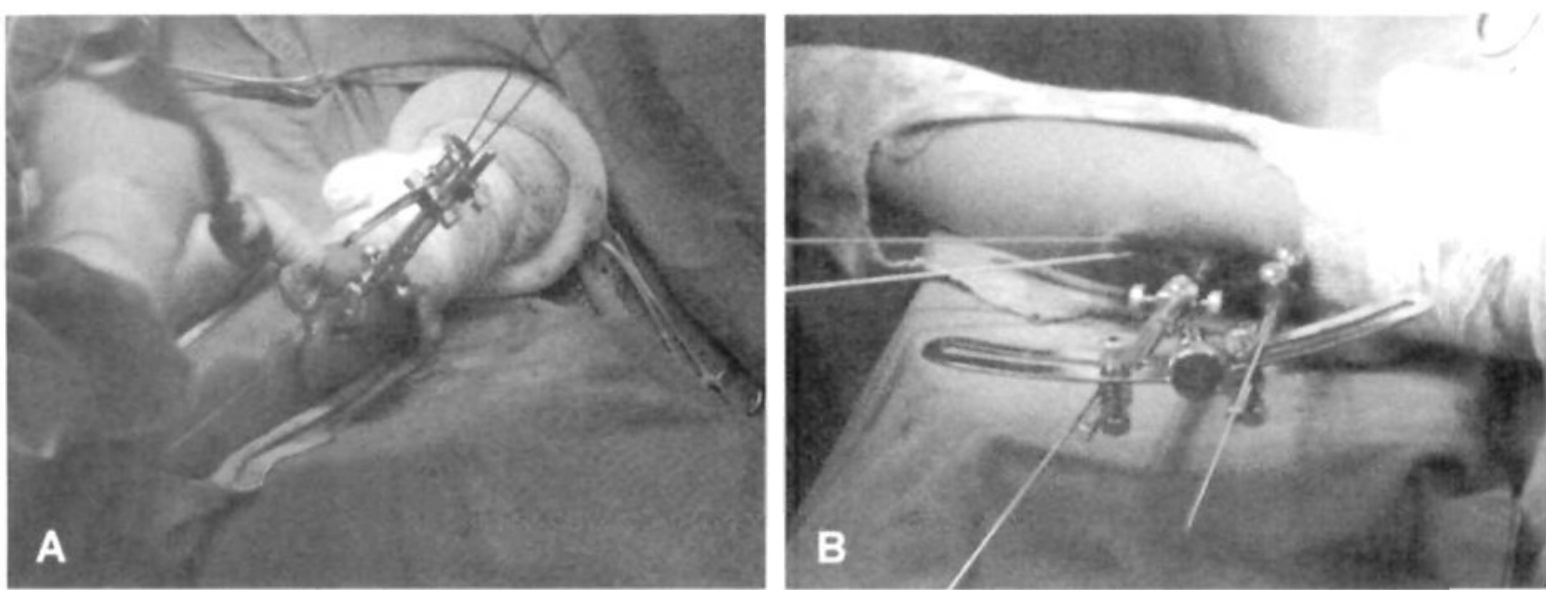

Fig. 6A-Application of Kirschner wire fixation is feasible without additional assistance. 6B-View of the surgical field after fixation.

ily, and the operation can be carried out efficiently with less radiation exposure to both the patients and the clinical staff.

Several surgical tools have been developed for corrective osteotomies, however, they are mainly focusing on the proximal tibia and femur[16-19], and scarcely over the humeral supracondyle. Application of computer assisted preoperative planning has been started for decades, and thorough discussions have been reported in other literatures[20-22]. The major advantages of using the CAD/CAM technologies lies on that not only can the design be modified easily, but also be tested virtually before being manufactured, which is both efficient and economic. After understanding the needs of the surgeons, several different designs have been developed and modified. Finally, a novel measuring fixator was designed mainly focusing

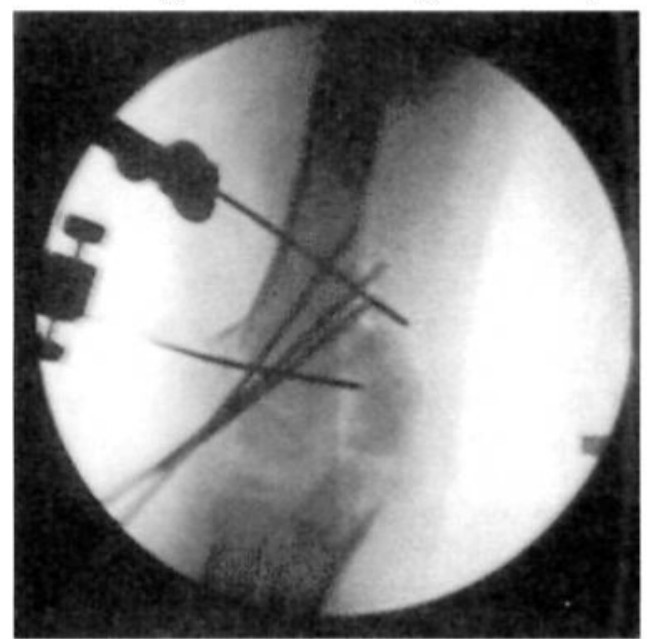

Fig. 7 Fluoroscopic reconfirmation of the osteotomy. A single Kirschner wire on each arm was able to provide sufficient stability in this case. on the simplicity of the instrument, and the ease of application, as Coyler suggested in his literature for the proximal femoral osteotomy[23].

Rapid prototype models are initially used in the field of industry as an fast and simple method to obtain the physical shape of the designed model, and has recently been applied in the medical field in several ways. In this study, several trials were performed over the rapid prototyping models of humerus to familiar with the use of the device before performing on the clinical cases. It is found that these physical replica models are helpful not only in the way that the supply is unlimited, but different deformities can be accurately produced. In addition, a real osteotomy was performed on a piece of adult cadaveric humeral bone. Although this instrument was originally designed for use in the pediatric elbow, we experienced even a good stability on the adult bone.

In addition to the proposed closing wedge osteotomy, it is believed that this instrument is also suitable for opening wedge osteotomy, and the surgical site is not limited to the supracondylar area, but other lesion sites as well. Further applications of this device will be carried out in the future.

\section{CONCLUSION}

Few devices have been reported in assistance for the humeral supracondylar osteotomy in children, yet such surgeries are not uncommon in daily practice for an orthpaedic surgeon. With the aid of computer $\mathrm{CAD} / \mathrm{CAM}$ technology, an innovative measuring fixator was designed and produced. Rapid prototyping technologies was applied to produce physical replica models of humerus for pre-operational reharsal. Two clinical trails was performed using proposed instrument with a positive results. This is another indica- 
tion of computer application in the orthopedic surgery, and with the integration of robotics technologies, computer-assistive orthopedic surgery (CAOS) will very soon be in sight.

\section{REFERENCES}

1. Graham B, et al. Supracondylar Osteotomy of the Humerus for Correction of Cubitus Varus. J Ped Orthop, 1990; 10:228-231.

2. Ippolito, et al. Post-traumatic cubitus varus. $J$ Bone and Joint Surg, 1990; 72(5)A: 757-765.

3. Hernandez MA and Roach JW. Corrective Osteotomy for Cubitus Varus Deformity. J of Ped Ortho, $1994 ; 14: 487-491$.

4. D'Ambrosia RD. Supracondylar fractures of the humerus: prevention of cubitus varus. J Bone Joint Surg, 1980; 61-A:46.

5. DeRosa GP, Graziano GP. A new osteotomy for cubitus varus. Clin Orthop Related Research, 1988; 236: 160-165.

6. Gaston P, et al, Supracondylar fracture of the humerus in children, Clin Orthop Related Research, 1993; 297:231-237.

7. Brewster AH, Karp M. Fractures in the region of the elbow in children: end result study. Surg Gynecol Obstet, 1978; 71:643-9.

8. Wilkins KE, Beaty JH, Chambers HG. Fractures and Dislocations of the Elbow Region. Rockwood and Green's Fractures in Adults. Vol 3. $4^{\text {th }}$ ed. Philadelphia: Lippincott-Raven Publishers; 1996; p.653-904.

9. Gaddy BC, et al. Distal Humeral Osteotomy for Correction of Posttraumatic Cubits Varus. $J$ Ped Orthop, 1994; 14:214-219.

10.Matshushita T, Nagano A. Arc Osteotomy of the Humerus to Correct Cubitus Varus. Clin Orthop Rel Reseasrch, 1997; 336:111-115.

11. Southwick WO. Osteotomy through the Lesser Trochanter for Slipped Capital Femoral Epiphysis. $J$ Bone Joint Surg (Am). 1967; 49:807-835.

12. Axer A. Subtrochanteric Osteotomy in the Treat- ment of Perthes' Disease. $J$ Bone Joint Surg (Br). $1965 ; 47: 489-499$.

13. French, PR. Varus deformity of the elbow following supracondylar fractures of the humerus in children. Lancet. 2:439, 1959.

14. Miniaci A, Ballmer FT, Ballmer PM, Jakob RP. Proximal Tibial Osteotomy-A New Fixation Device. Clin Ortho Rel Res. 1989; 246:250-259.

15. Merten HA, Wiese KG. Simple Device for Plate Positioning and Contouring. J Oral Maxillofac Surg. 1995; 53:625-626.

16. Ito H, Minami A, Suzuki K, Matsuno T. ThreeDimensionally Corrective External Fixator System for Proximal Femoral Osteotomy. $J$ Ped Ortho. 2001; 21:652-656.

17. Minns RJ, Epstein HP, Porter BB. Tools for High Tibial Wedge Osteotomy. J Biomed Eng. 1985; 7:256-258.

18. Takahashi $T$, Wada $Y$, Tanaka $M$, Iwagawa $M$, Ikeuchi M, Hirose D, et al. Dome-Shaped Proximal Tibial Osteotomy using Perfutaneous Drilling for Osteoarthritis of the Knee. Arch Orthop Truma Surg. 2000; 120:32-37.

19. Mizuseki T, Tsuge K, Ikuta Y. Precise UlnaShortening Osteotomy with a New Device. $J$ of Hand Surgery-American Volume. 2001; 26 (5):931939.

20. Tsai MD, Hsieh MS, Hou SB. Virtual Reality Orthopedic Surgery Simulator. Computers in Biology and Medicine. 2001; 31:333-351.

21. Lee MY, Chang CC, Lin CC, Lo LJ, Chen YR. 3D Image Reconstruction and Rapid Prototyping Models in Custom Implant Design for patient with Cranial Defect. IEEE

22. Croitoru H, Ellis RE, Prihar R, Small CF, Pichora DR. Fixation-Based Surgery: A New Technique for Distal Radius Osteotomy. Computer Aided Surgery. 2001; 6: 160-169.

23. Coyler RA. Compression External Fixation after Biplane Femoral Trochanteric Osteotomy for Severe Slipped Capital Femoral Epiphysis. $J$ Bone Joint Surg (Am). 1980;62:557-560. 